\title{
АЛГОРИТМЫ ОБРАБОТКИ СЕЙСМОРАЗВЕДОЧНЫХ ДАННЫХ ДЛЯ ПОВЫШЕНИЯ ДОСТОВЕРНОСТИ РЕЗУЛЬТАТОВ СКОРОСТНОГО АНАЛИЗА
}

\author{
М.В. Тарантин \\ Горный институт УрО РАН, г. Пермь
}

\begin{abstract}
Аннотация. Предложена процедура временной регуляризации скоростных характеристик, получаемых при непрерывном скоростном анализе материалов шахтной сейсморазведки. Процедура основана на статистическом анализе и последующей фильтрации значений скоростей суммирования. На реальных данных рассмотрены варианты обработки скоростей суммирования для получения физически непротиворечивых результатов. Проведен сравнительный анализ результатов построения временных разрезов с применением новой процедуры и некоторых известных.
\end{abstract}

Ключевые слова: сейсмическая разведка, скоростной анализ, отраженные волны, статистический анализ.

Скоростной анализ сейсмических данных позволяет определить распределение скоростей упругих волн в изучаемой среде, и на основании этой информации интерпретировать зарегистрированные сейсмограммы, преобразуя их в суммотрассы и временной разрез. Многообразие методов такой обработки можно разделить на две большие группы: ручной подбор наиболее подходящих зависимостей скорости от глубины и автоматизированный. Преимущество первой группы в достоверности результатов, второй - в скорости их получения. В обоих случаях критерием качества служит степень регулярности волновой картины, математически обычно выражаемая функцией когерентности. Различия результатов обусловливаются стратегией отбраковки подходящих вариантов: при ручной обработке отмечаются несколько наиболее контрастных сигналов отражений, при машинном анализе их может быть существенно больше. Проблема состоит в адекватной реальной геологической ситуации увязке найденных значений между соседними точками профиля и по глубине после машинной обработки.

Один из вариантов приближения результатов непрерывного машинного анализа к ручному варианту, когда значимыми считаются наиболее контрастные отражения, представлен в работе [1]. Однако этот алгоритм способен пропустить малые колебания скоростных свойств среды, что особенно актуально для шахтных исследований. Цель настоящей работы состоит в исследовании новой процедуры обработки данных непрерывного скоростного анализа [2] и сравнении ее результатов с другими имеющимися алгоритмами.

В качестве примера волнового поля использован массив записей, полученных в шахте одного из рудников в пределах Верхнекамского месторождения калийных солей. Источники и приемники упругих колебаний располагались в выработке в пределах калийной залежи, причем источники колебаний направлены в кровлю выработки вертикально вверх, а приемники расположены в подошве выработки также вертикально. При такой системе наблюдений возможно изучение продольных и поперечных волн, распространяющихся преимущественно в верхнем относительно выработки полупространстве [3], что уменьшает неопределенности при интерпретации.

На рис. 1 представлены результаты предварительной обработки волновых материалов. Исходные сейсмограммы (1a) подвергаются частотной фильтрации и процедуре выравнивания амплитуд (1б) с целью выделения целевых отраженных волн. Для выделения информативных, в данном случае поперечных, волн применен подход, основан- 
ный на различии скоростей продольных и поперечных волн [4], в результате на сейсмограмме остаются преимущественно целевые годографы (1в). Видимые на больших временах наклонные оси синфазности, представляющие в данном случае помеху, подавлены (1г) в пространстве волновых чисел после пересортировки трасс, как описано в работе [5].

Для сравнения использовано 4 варианта кинематических поправок, позволяющих построить суммотрассы и в итоге временной разрез: рассчитанные по одному среднему скоростному закону, рассчитанные по двум выбранным вручную в относительно однородных интервалах профиля скоростным законам, рассчитанные по результатам непрерывного скоростного анализа профиля и вычисленные после предлагаемой регуляризирующей обработки результатов непрерывного анализа.

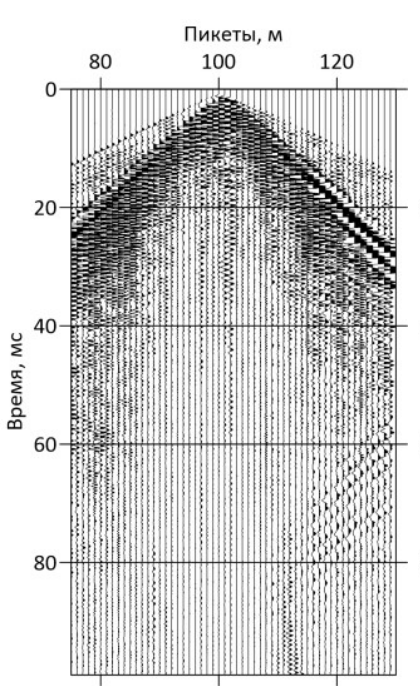

a



6



B

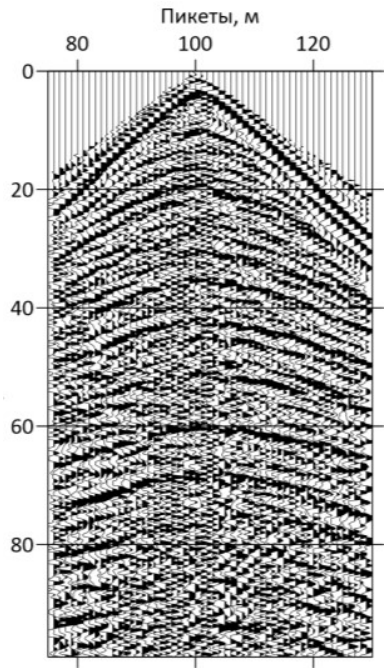

$\Gamma$

Рис. 1. Предварительная обработка сейсморазведочных данных. Пояснения в тексте

Для первого варианта скоростной закон выбран постоянным, не зависящим от времени, так как в пределах исследуемого интервала соляных пород такое приближение вполне оправданно - за среднюю скорость поперечных волн принято значение 2400 м/с. Для второго варианта скоростные законы определены вручную для интервала пикетов в окрестности одной и трех четвертей общей длины профиля, эти интервалы выбраны, исходя из имеющейся априорной информации, как наиболее однородные и представительные. Для непрерывной обработки профиля использована процедура построения суперсейсмограммы с базой, сравнимой с длиной расстановки при наблюдениях. Это понижает влияние шумов и повышает достоверность определяемых скоростей. Регуляризация полученных данных проводилась со следующими параметрами: ширина временного окна анализа скоростей вдвое превышает использованное для построения когерентности окно, предельное значение отклонения от среднего равно среднеквадратическому в окне.

Различие скоростных кривых можно оценить по примеру на рис. 2. Непрерывное сканирование дает кривую (3), которая оказывается дифференцирована по глубине настолько, что не поддается обоснованной интерпретации; кроме того, она имеет недостоверные значения, совпадающие с краями допустимого интервала анализа. После удаления краевых значений область вероятных величин скорости (6) сужается, что позволяет определить результирующую (8) достаточно гладкую кривую. В данном случае она близка к исходной (1), но отражает вероятные локальные особенности строения среды. 


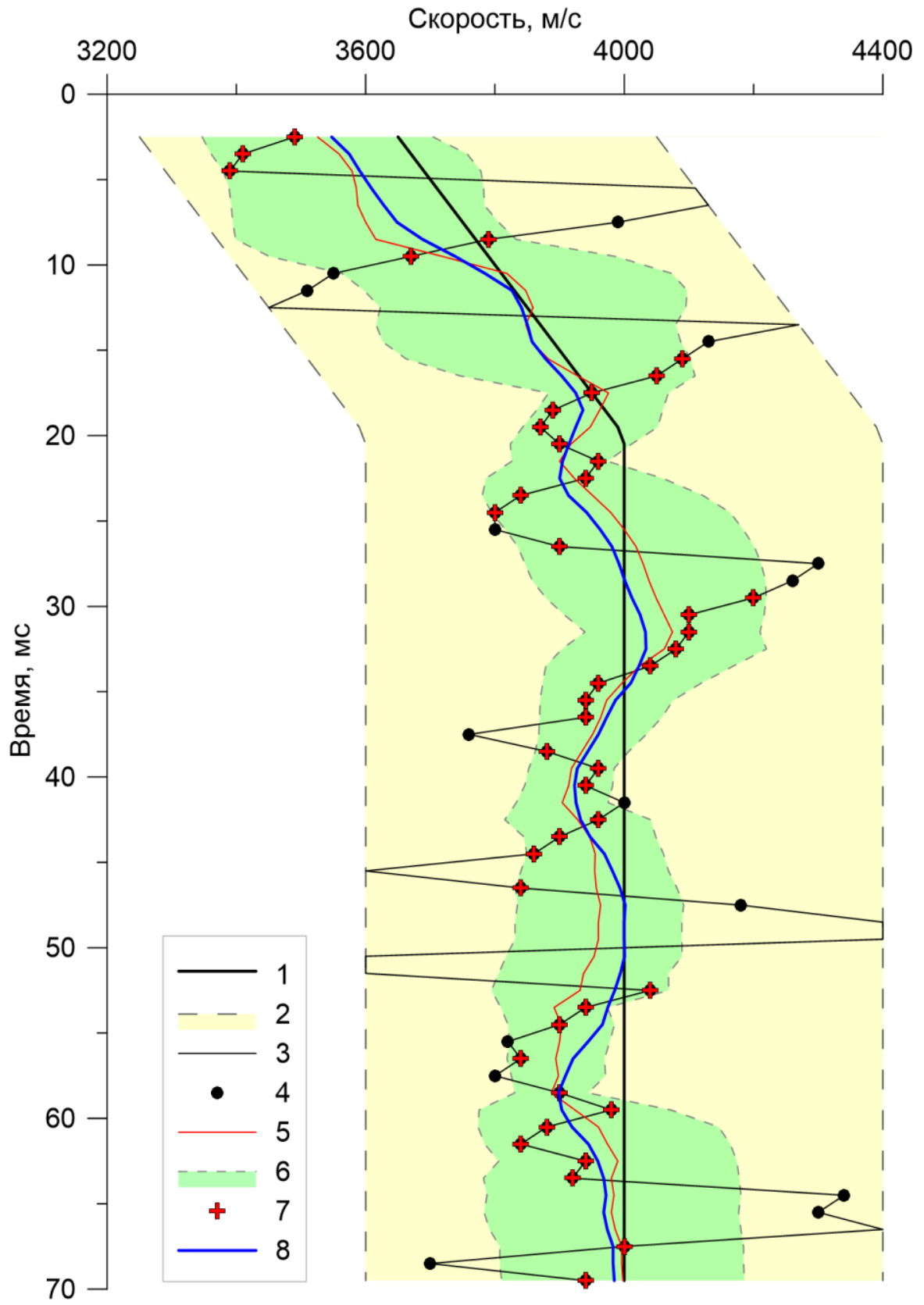

Рис. 2. Обработка скоростных характеристик статистическим методом

1 - априорный скоростной закон, 2 - границы допустимого интервала при поиске скоростей,

3 - результаты вычислений скорости методом максимального значения когерентности,

4 - то же, после отсеивания краевых значений, 5 - кривая усредненных в скользящем окне значений 4 , 6 - интервал допустимых отклонений от среднего значения (ширина - 1,0 б), 7 - значения скорости, попавшие в доверительный интервал 6,8 - результирующая кривая скоростного закона

Фрагменты результирующих разрезов приведены на рис. 3. Несмотря на относительную скоростную однородность горных пород, два (б) и более (в, г) опорных скоростных закона лучше выделяют отражения, чем один средний (а). Можно отметить чрезвычайную похожесть вариантов (в) и (г), однако скоростные характеристики, лежащие в их основе, различны (рис. 4).

Дополнительные исследования показали, что полученные после процедуры результаты обладают устойчивостью в том смысле, что повторная обработка с теми же параметрами практически не изменяет данные. Для заметных изменений необходимо изменить либо доверительный интервал, либо окно анализа. 


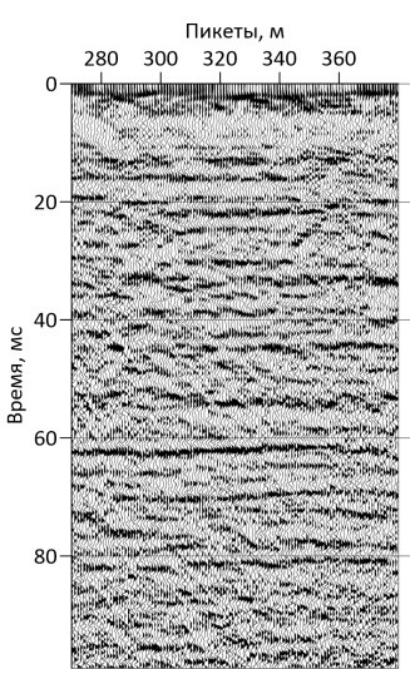

a
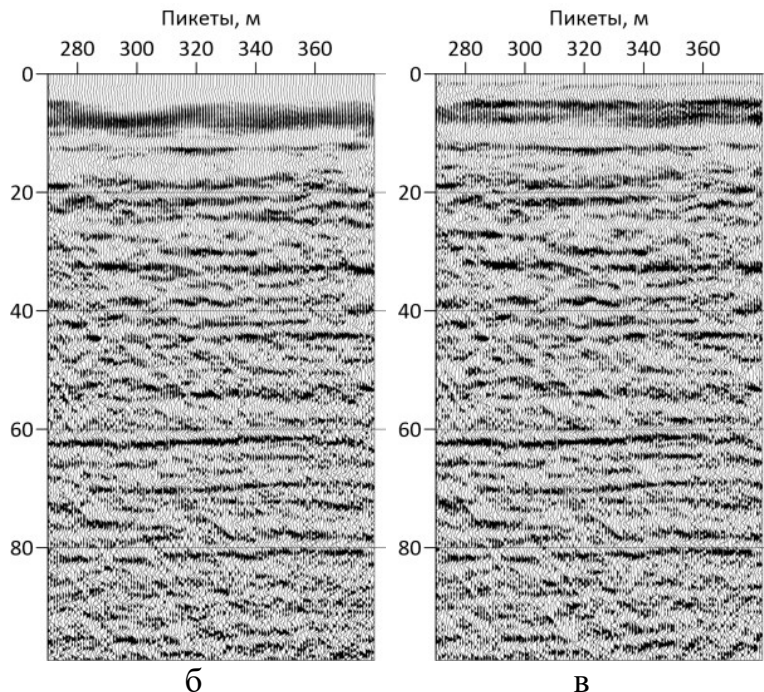

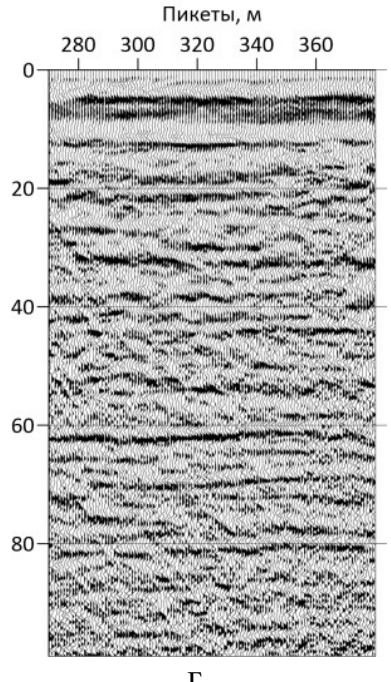

Рис. 3. Результаты построения временного разреза при различных кинематических поправках: один средний скоростной закон - a, два закона для каждого из концов профиля - б, непрерывный скоростной анализ - в, обработанные по предлагаемому алгоритму данные - г
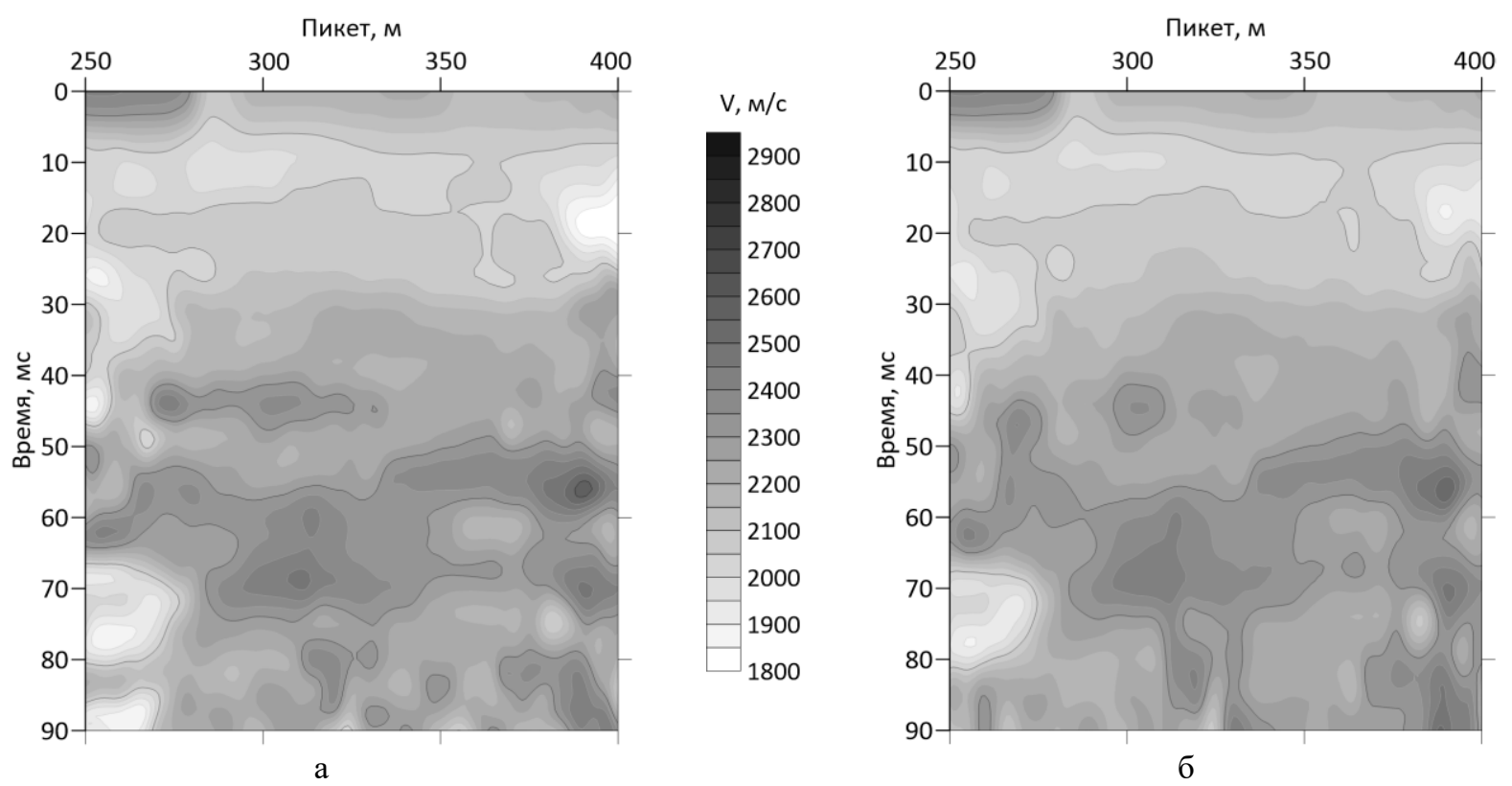

Рис. 4. Скоростные характеристики до (а) и после (б) регуляризации кривых

Работа выполнена при поддержке РФФИ (грант № 19-45-590015 p_a).

\section{БИБЛИОГРАФИЧЕСКИЙ СПИСОК}

1. Тарантин М.В., Ярославцев А.Г. Учет латеральной изменчивости скоростей при цифровой обработке данных малоглубинной сейсморазведки // Горное эхо. - 2018. - № 1-2 (70-71). - С. 88-91.

2. Ярославцев А.Г., Тарантин М.В., Байбакова Т.В. Повышение достоверности результатов скоростного анализа при малоглубинной сейсморазведке // Инженерная и рудная геофизика - 2021: 17-я науч.практ. конф. и выставка. - Геленджик, 2021. - (в печати).

3. Ярославцев А.Г., Клестов И.М. Особенности обработки данных шахтной сейсморазведки с целью разделения Р и $\mathrm{S}$ волн // Горное эхо. - 2020. - № 3 (80). - C. 101-106. - DOI: 10.7242/echo.2020.3.20. 
4. Ярославцев А.Г., Фатькин К.Б. Шахтные сейсмоакустические исследования при контроле предохранительных целиков в калийных рудниках //. Инженерная и рудная геофизика -2020 : 16-я науч.-практ. конф. и выставка, 14-18 сент. 2020. - Пермь, 2020. - DOI: 10.3997/22144609.202051043.

5. Бобров В.Ю. Оптимальные пути подавления боковых волн-помех при цифровой обработке шахтных сейсмоакустических данных // Горное эхо. - 2021. - № 1 (82). - С. 68-74.

УДК 550.8.052

DOI:10.7242/echo.2021.2.13

\title{
МЕТОД РЕДКИХ СОЧЕТАНИЙ ПРИ ВЫДЕЛЕНИИ ЗОН ИНЖЕНЕРНО-ГЕОЛОГИЧЕСКИХ ОСЛОЖНЕНИЙ ПО ЭЛЕКТРОРАЗВЕДОЧНЫМ ДАННЫМ
}

\author{
Л.А. Христенко \\ Горный институт УрО РАН, г. Пермь
}

\begin{abstract}
Аннотация: Для повышения достоверности интерпретации электроразведочных наблюдений методами естественного поля и симметричного электропрофилирования, выполненного на трех разносах питающей линии, предложено использовать метод редких сочетаний. Метод основан на расчете суммарных матриц относительных частот встречаемости различных параметров. Минимальные значения параметра МРС (суммарные частости) отвечают аномальным - редким сочетаниям всех использованных признаков. Метод был опробован на участке, расположенном в пределах шахтного поля СКРУ-2 Верхнекамского месторождения солей. Исходными признаками для вычисления параметров МРС служили статистические характеристики электрических параметров (значений потенциала ЕП и значений кажущегося сопротивления). Сравнение результатов интерпретации, полученных с использованием параметров МРС и результатов классификаций методом общего расстояния, между собой показало, что области предполагаемых инженерно-геологических осложнений устойчиво выделяются двумя методами, имеющими различный математический аппарат. Т.е. каждый из методов повышает достоверность выделенных осложнений природного или техногенного происхождения.
\end{abstract}

Ключевые слова: кажущееся сопротивление, потенциал естественного поля, статистические характеристики, метод редких сочетаний.

Интерпретация данных электроразведочных методов естественного поля (ЕП) и симметричного электропрофилирования (СЭП), входящих в комплекс методов, используемых при решении различных геологических и горнотехнических задач, связанных с обеспечением эффективности и безопасности отработки соляных месторождений, чаще всего основана на простейших приемах оценки глубин залегания и размеров источников или на качественном анализе. Такие приемы интерпретации приводят к неоднозначным выводам. Достоверность и информативность интерпретации можно повысить, используя различные преобразования электрометрических параметров. Преобразования позволяют более четко проследить неявно выраженные в наблюденных полях особенности геологического строения. Результаты использования процедур безэталонной классификации, быстрого вейвлет-преобразования дискретных значений кажущегося сопротивления (КС); методы эмпирической модой декомпозиции (EMD) уже были подробно представлены ранее $[4,5,10,11]$. В [9] дана краткая ретроспектива определения эффективного набора процедур преобразования данных ЕП и СЭП. Кроме того, рассмотрен еще один метод безэталонного прогнозирования или эвристический метод выделения аномальных зон по комплексу признаков - метод редких сочетаний (МРС), впервые предложенный в 1973 году Н.Н. Боровко [1].

Суть метода состоит в том, что в каждой точке задания поля выполняется расчет относительной частоты его встречаемости, рассчитанной как отношение числа зна- 